\title{
A critical reassessment of the fundamental properties of GJ 504: chemical composition and age ${ }^{\star}$
}

\author{
V. D’Orazi ${ }^{1,2,3}$, S. Desidera ${ }^{1}$, R. G. Gratton ${ }^{1}$, A. F. Lanza ${ }^{4}$, S. Messina ${ }^{4}$, S. M. Andrievsky ${ }^{5,6}$, S. Korotin ${ }^{5,7}$, \\ S. Benatti ${ }^{1}$, M. Bonnefoy ${ }^{8,9}$, E. Covino ${ }^{10}$, and M. Janson ${ }^{11}$ \\ ${ }^{1}$ INAF Osservatorio Astronomico di Padova, vicolo dell'Osservatorio 5, 35122 Padova, Italy \\ e-mail: valentina.dorazi@oapd.inaf.it \\ 2 Department of Physics and Astronomy, Macquarie University, Sydney, NSW 2109, Australia \\ 3 Monash Centre for Astrophysics, School of Physics and Astronomy, Monash University, Melbourne, VIC 3800, Australia \\ ${ }^{4}$ INAF Osservatorio Astrofisico di Catania, via S. Sofia 78, 95123 Catania, Italy \\ 5 Department of Astronomy and Astronomical Observatory, Odessa National University, Isaac Newton Institute of Chile, \\ Odessa Branch, Shevchenko Park, 65014 Odessa, Ukraine \\ ${ }^{6}$ GEPI, Observatoire de Paris, PSL Research University, CNRS, Université Paris Diderot, Sorbonne Paris Cité, Place Jules Janssen, \\ 92195 Meudon, France \\ 7 Crimean Astrophysical Observatory, 298409 Nauchny, Crimea \\ 8 Univ. Grenoble Alpes, IPAG, 38000 Grenoble, France \\ 9 CNRS, IPAG, 38000 Grenoble, France \\ ${ }^{10}$ INAF Osservatorio Astronomico di Capodimonte, Salita Moiariello 16, 80131 Napoli, Italy \\ 11 Stockholm University, AlbaNova University Center, 10691 Stockholm, Sweden
}

Received 11 July 2016 / Accepted 7 September 2016

\begin{abstract}
Context. The recent development of brand new observational techniques and theoretical models have greatly advanced the exoplanet research field. Despite significant achievements, which have allowed the detection of thousands extrasolar systems, a comprehensive understanding of planetary formation and evolution mechanisms is still desired. One relevant limitation is given by the accuracy in the measurements of planet-host star ages. The star GJ 504 has been found to host a substellar companion whose nature is strongly debated. There has been a recent difference of opinion in the literature owing to the uncertainty on the age of the system: a young age of $\sim 160 \mathrm{Myr}$ would imply a giant planet as a companion, but a recent revision pointing to a solar age ( 4 Gyr) instead suggests a brown dwarf.

Aims. With the aim of shedding light on this debated topic, we have carried out a high-resolution spectroscopic study of GJ 504 to derive stellar parameters, metallicity, and abundances of both light and heavy elements, providing a full chemical characterisation. The main objective is to infer clues on the evolutionary stage (hence the age) of this system.

Methods. We performed a strictly differential (line-by-line) analysis of GJ 504 with respect to two reference stars, that is the planethost dwarf $\iota$ Hor and the subgiant HIP 84827. The former is crucial in this context because its stellar parameters (hence the evolutionary stage) is well constrained from asteroseismic observations. Regardless of the zero point offsets, our differential approach allows us to put tight constraints on the age of GJ 504 with respect to $\iota$ Hor, thereby minimising the internal uncertainties.

Results. We found that the surface gravity of GJ 504 is $0.2 \pm 0.07$ dex lower than that of the main-sequence star $\iota$ Hor, suggesting a past turn-off evolution for our target. The isochrone comparison provides us with an age range between 1.8 and $3.5 \mathrm{Gyr}$, with a most probable age of $\approx 2.5 \mathrm{Gyr}$. Thus, our findings support an old age for the system; further evidence comes from the barium abundance, which is compatible with a solar pattern and not enhanced as observed in young stars.

Conclusions. We envisaged a possible engulfment scenario to reconcile all the age indicators (spectroscopy, isochrones, rotation, and activity); this engulfment could have occurred very recently and could be responsible for the enhanced levels of rotation and chromospheric activity, as previously suggested. We tested this hypothesis, exploiting a tidal evolution code and finding that the engulfment of a hot Jupiter, with mass not larger than $\approx 3 M_{\mathrm{j}}$ and initially located at $\approx 0.03 \mathrm{AU}$, seems to be a very likely scenario.
\end{abstract}

Key words. stars: abundances - stars: individual: GJ 504 - stars: fundamental parameters - stars: solar-type - stars: evolution

\section{Introduction}

Hunting for planetary systems outside our solar system, with the ultimate goal of revealing habitable worlds, is undoubtedly one of the most intriguing and fascinating topics in mod-

* Based on observations made with ESO Telescopes at the La Silla Paranal Observatory under programme ID 072.A-9006(A) and 083.A-9003(A). ern astrophysics. Outstanding efforts have been put forwards to detect (and characterise) extrasolar systems, such that 5437 planet candidates have been identified so far ${ }^{1}$. The presence of those systems has been mostly inferred via indirect methods (e.g. radial velocity variations or transits). However, direct imaging techniques are starting to provide us with powerful and complementary tools to detect planetary

\footnotetext{
1 Source http: //exoplanets . eu, as for July 2016.
} 
companions, thanks to the advent of new-generation instruments purposely designed for this goal, such as GPI, ScEXAO, and SPHERE (see Macintosh et al. 2014; Martinache \& Guyon 2009; Beuzit et al. 2008, respectively). Unfortunately, beyond the technical limitations, which are nowadays primarily due to adaptive optics performance, several other fundamental issues affect our comprehension of the substellar regime, preventing us from gathering extensive knowledge. Along with several controversies related to, for instance, planetary atmosphere models, evolutionary stages of substellar objects, different formation, and early evolution scenarios (core accretion versus disc instability, and/or hot-start versus cold-start models; Pollack et al. 1996; Kratter et al. 2010; Marley et al. 2007; Fortney et al. 2008), the major concern in characterising exoplanetary systems is represented by the uncertainty on the stellar age. The age estimate of the planet-host star dramatically impacts the inferred mass for substellar companions and hence the calibration of the ageluminosity relationship for substellar objects. Thus, it is crucial to our understanding of how planets have formed.

Expanding upon our previous work on GJ 758 (see Vigan et al. 2016), in this paper we present a similar investigation focussing on the curious case of GJ 504. The age of this star is hotly debated. The short rotational period of $P_{\text {rot }}=3.3$ days (Donahue et al. 1996) and the pronounced level of chromospheric and coronal activities seem to suggest a young age for the system, that is $160_{-60}^{+70} \mathrm{Myr}$ from gyrochronology and, from the chromospheric level, slightly older at $330 \pm 180 \mathrm{Myr}$, as traced by the $\mathrm{Ca}$ II $\mathrm{H}$ and $\mathrm{K}$ emission. Assuming the young age of $160 \mathrm{Myr}$ and spectroscopic parameters as published by Valenti \& Fischer (2005), Kuzuhara et al. (2013) concluded that GJ 504 is a young, main-sequence star with $T_{\text {eff }} \approx 6200 \mathrm{~K}$ and $\log g=4.6 \mathrm{dex}$ (with $g$ in $\mathrm{cm} \mathrm{s}^{-2}$ ). These authors were able to claim that the substellar companion orbiting at 43.5 AU, discovered through direct imaging observations within the framework of the SEEDS survey, has an inferred mass of $4_{-1.0}^{+4.5} M_{\text {Jup }}$. This is the lowest mass companion ever directly imaged to date. However, Fuhrmann \& Chini (2015) questioned this finding, arguing that the star is actually much older and is comparable with a turn-off stage of evolution and a solar-like age. In addition, these authors envisaged a possible merging scenario, with a now engulfed substellar object, to account for the enhanced level of activity and rotation. This study suggested an upward revision for the companion mass, implying that we are dealing with a brown dwarf $\left(M \approx 30-40 M_{\text {Jup }}\right)$ rather than a giant planet. An additional motivation for the interest in the GJ 504 system is derived by Skemer et al. (2016), who found an indication that the substellar companion is more enriched $([\mathrm{M} / \mathrm{H}] \approx+0.5)$ in metal than its parent $\operatorname{star}([\mathrm{M} / \mathrm{H}] \approx+0.1-0.3)$.

We carried out a strictly differential analysis of GJ 504 with respect to three other reference stars ( $\iota$ Horologii, HIP 84827, and the Sun) to shed light on this complicated picture, by obtaining in this way very accurate spectroscopic parameters and abundances. We derived temperature, gravity, metallicity, and several key species abundances, from the light Li and $\mathrm{C}$ up to elements synthesised via neutron-capture reactions $(\mathrm{Ba})$. The manuscript is organised as follows: in Sect. 2.2 we present the spectroscopic observations and abundance analysis procedure and results. In Sect. 3 we extensively discuss all the other age indicators, whereas we sketch a plausible engulfment scenario to account for all the observed properties in Sect. 4. We stress that in the present work we are focussing on the fundamental properties of the parent star. The complete characterisation of the substellar companion GJ 504b will be published in a companion paper (Bonnefoy et al., in prep.).

\section{Spectroscopic parameters and elemental abundances}

\subsection{Observations and analysis}

We exploited high-resolution (nominal resolution $R=48000$ ), high signal-to-noise FEROS (Kaufer et al. 1999) spectra for all the stars under scrutiny. The observations were carried out during nights in December 2003 and April 2009 and the original data were retrieved through the ESO archive. Data reduction was performed using a modified version of the FEROS-DRS pipeline (running under the ESO-MIDAS context FEROS), yielding the wavelength-calibrated spectrum that is merged and normalised through standard steps. The spectra provide an almost complete coverage from $3500 \AA$ to $9200 \AA$ with $\mathrm{S} / \mathrm{N}$ between 300 and 500 at $6700 \AA$ for the three stars; Desidera et al. (2015) provides further details. Along with GJ 504, we analysed $\iota$ Hor and HIP 84827 as reference stars. The former is a planethost, main-sequence star that is relatively young $(\approx 600 \mathrm{Myr})$ and slightly metal rich $([\mathrm{Fe} / \mathrm{H}] \approx 0.15-0.20 \mathrm{dex})$. It is suspected to be an evaporated member from the Hyades supercluster (Vauclair et al. 2008; Biazzo et al. 2012). The activity level and projected rotational velocity of $\iota$ Hor are similar to those of GJ 504, ensuring minimal impact of line blending and possible differences in the atmospheric structure of active stars (Santos et al. 2008; D’Orazi \& Randich 2009) when performing a differential abundance analysis. Conversely, HIP 84827 is an evolved, chromospherically quiet star with metallicity around +0.2 dex and gravity in agreement with a post turn-off evolution (e.g. Ramírez et al. 2014). Given the effective temperature, $T_{\text {eff }}$ around $6000 \mathrm{~K}$, and the metal-rich nature of $\iota$ Hor and HIP 84827, they are much more similar in terms of atmospheric structural properties to GJ 504 than to the Sun; for this reason, we selected these stars as reference frames for our strictly differential analysis. For sake of completeness, we also analysed a solar spectrum acquired with the same instrument to furnish absolute abundances. The solar values obtained within the present study for species from $\mathrm{Li}$ to $\mathrm{Ba}$ are reported in Table 1; we adopted $T_{\text {eff }, \odot}=5777 \mathrm{~K}, \log g_{\odot}=4.44 \mathrm{dex}, \xi_{\odot}=0.88 \mathrm{~km} \mathrm{~s}^{-1}$, and $A(\mathrm{Fe})_{\odot}=7.50$ dex.

We conducted abundance analyses through equivalent width (EW) measurements for all species but $\mathrm{Li}, \mathrm{K}$, and $\mathrm{Ba}$, for which we instead performed spectral synthesis computation. We measured the EWs with the ARES code (Sousa et al. 2007), but we rigorously double checked every single line with the IRAF ${ }^{2}$ task splot with special care to the bluest wavelength domain $(\lambda \lambda \lesssim$ $5000 \AA)$, where the severe crowding hampers a straightforward definition of the continuum. Our line-list contains a grand total of 136 and 18 lines for Fe I and Fe II, respectively, and it is available upon request. For other species we instead selected spectral lines (adopting corresponding atomic parameters) from Meléndez et al. (2014). We derived LTE abundances using MOOG by Sneden (1973, 2014 version) and employing the Kurucz grid of model atmospheres (Castelli \& Kurucz 2004) with solar-scaled chemical composition and no overshooting. We obtained both stellar parameters and elemental abundances by means of a differential line-by-line analysis for GJ 504 with respect to $\iota$ Hor and HIP 84827 . The first step consists in the determination of the atmospheric parameters and metallicity by means of the spectroscopic analysis, following the standard procedure. Effective temperatures ( $\left.T_{\text {eff }}\right)$ were derived by zeroing the

2 IRAF is the Image Reduction and Analysis Facility, a general purpose software system for the reduction and analysis of astronomical data. IRAF is written and supported by National Optical Astronomy. 
Table 1. Solar abundances from the present study and from Grevesse et al. (1996), Asplund et al. (2009), and meteoritic abundances from Lodders et al. 2009 ( $\mathrm{G}+96, \mathrm{~A}+09$, and $\mathrm{L}+09$, respectively).

\begin{tabular}{lcccc}
\hline \hline Species & This work & G+96 & A+09 & L+09 \\
\hline $\mathrm{Li}$ & 1.05 & 1.16 & 1.05 & 3.26 \\
$\mathrm{C}$ & 8.41 & 8.55 & 8.43 & 7.39 \\
$\mathrm{O}$ & 8.63 & 8.97 & 8.69 & 8.40 \\
$\mathrm{Na}$ & 6.23 & 6.33 & 6.24 & 6.27 \\
$\mathrm{Mg}$ & 7.58 & 7.58 & 7.60 & 7.53 \\
$\mathrm{Al}$ & 6.36 & 6.47 & 6.45 & 6.43 \\
$\mathrm{Si}$ & 7.50 & 7.55 & 7.51 & 7.51 \\
$\mathrm{~S}$ & 7.11 & 7.33 & 7.12 & 7.15 \\
$\mathrm{~K}$ & 5.07 & 5.12 & 5.03 & 5.08 \\
$\mathrm{Ca}$ & 6.30 & 6.36 & 6.34 & 6.29 \\
$\mathrm{Ti}$ & 4.92 & 5.02 & 4.95 & 4.91 \\
$\mathrm{Cr}$ & 5.59 & 5.67 & 5.64 & 5.64 \\
$\mathrm{Fe}$ & 7.50 & 7.50 & 7.50 & 7.45 \\
$\mathrm{Ni}$ & 6.22 & 6.25 & 6.22 & 6.20 \\
$\mathrm{Zn}$ & 4.61 & 4.60 & 4.56 & 4.63 \\
$\mathrm{Ba}$ & 2.17 & 2.13 & 2.18 & 2.18 \\
\hline
\end{tabular}

slope between differential abundances from Fe I lines and the excitation potential of the spectral features. Similarly, microturbulence values $(\xi)$ were obtained imposing no spurious trends between differential abundances from Fe I and the reduced EWs $(E W / \lambda)$. Surface gravities $(\log g)$ instead come from the ionisation balance, that is $\langle\Delta(\mathrm{FeII})-\Delta(\mathrm{FeI})\rangle=0$. The solution is reached when all the three conditions are simultaneously satisfied better than $1 \sigma$ from the error on slopes for temperature and microturbulence and better than roughly one-third the error bar in FeI and FeII features (i.e. the standard deviation from the mean), as previously carried out by Meléndez et al. (2014). We show in Fig. 1 differential abundances of GJ 504 with respect to $\iota$ Hor as a function of the excitation potential (upper panel) and the reduced EW (lower panel). The gravity optimised by using $\mathrm{Fe}$ lines also satisfies the ionisation balance for $\mathrm{Cr}$ and $\mathrm{Ti}$, within the observational uncertainties, corroborating our gravity estimate. This is evident from Fig. 2, where abundances from neutral and singly ionised lines are shown for $\mathrm{Cr}$, $\mathrm{Ti}$, and $\mathrm{Fe}$. We performed this procedure twice and we assumed as final spectroscopic parameters for GJ 504 the average estimate, for $T_{\text {eff }}$, $\log g, \xi$, and $[\mathrm{Fe} / \mathrm{H}]$, with respect to $\iota$ Hor and HIP 84827 (see next section). Once atmospheric parameters and metallicity are inferred, we proceed to derive the other elemental abundances. Oxygen abundances, which come from the EW measurements of the strong permitted triplet at 7771-7775 $\AA$, were corrected for $3 \mathrm{D}$ effects and departures from the LTE, following prescriptions by Amarsi et al. $(2015)^{3}$.

As previously mentioned, abundances for $\mathrm{Li}, \mathrm{K}$, and $\mathrm{Ba}$ come from spectral synthesis, employing the driver synth in MOOG. As for Li we used the same list as in our previous works

\footnotetext{
3 http://inspect-stars.com
}
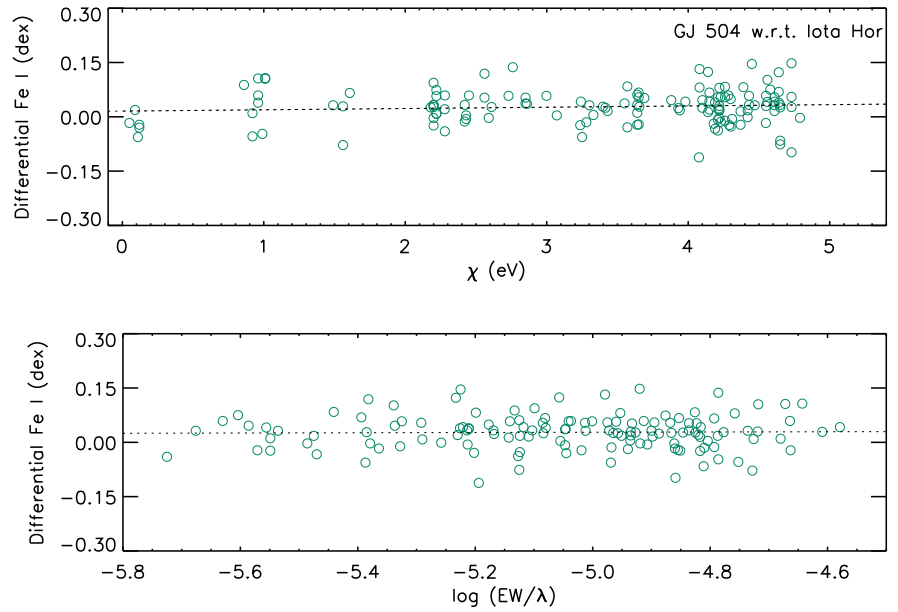

Fig. 1. Differential iron abundances vs. excitation potential (top panel) and reduced equivalent width (bottom panel) of Fe I lines. The dashed lines are linear fits to the $\mathrm{Fe} \mathrm{I}$ lines.
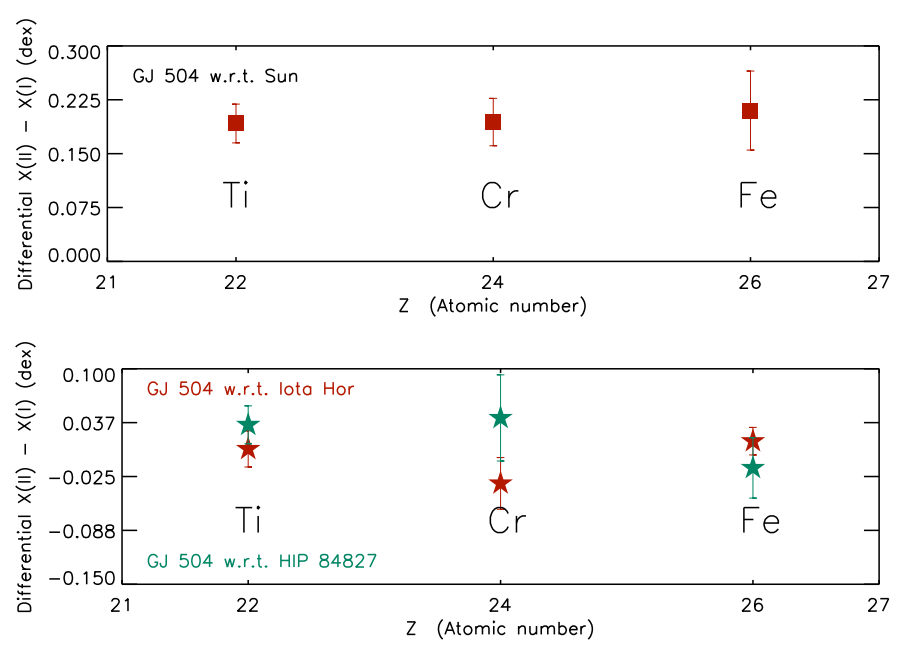

Fig. 2. Singly ionised minus neutral differential abundances of $\mathrm{Fe}, \mathrm{Cr}$, and Ti for differential analysis with respect to the solar spectrum (upper panel), ८ Hor and HIP 84827 (lower panel).

(e.g. Desidera et al. 2011), which covers from 6700 to $6720 \AA$, including the strong Li I doublet at $6707.78 \AA$. We calculated the best match between observed and synthetic spectra, changing the $\mathrm{Li}$ abundances and exploiting the $\mathrm{Ca}$ I line at $6717.69 \AA$ to evaluate the spectral broadening.

In our spectra only two K I lines are available for the quantitative analysis, that is the resonance doublet at 7665-7699 $\AA$. The first line is often blended with terrestrial bands. At the solar metallicity both lines have very strong wings and this prevents the accurate measurements of their equivalent widths. In this case the better way to analyse these lines is to use the synthetic spectrum method.

The non local thermodynamical equilibrium (NLTE) effects are rather strong in these lines (Bruls et al. 1992; Ivanova \& Shimanskii 2000). To take these effects into account, we used the atomic model that includes all transitions between the first 20 levels and the ground level of K II. This model was described in details in Andrievsky et al. (2010).

Barium abundances were derived by synthesing the two Ba II lines at $5853 \AA$ and $6141 \AA$, taking hyperfine structure and 
isotopic splitting into account (from McWilliam et al. 1995). The Ba II feature at $6141 \AA$ is known to be blended with a Fe I line, which is particularly strong in our case given the metal-rich nature of our objects. However, the spectral synthesis technique allows us to properly take into account this blend and the strictly differential analysis minimises this source of error. Also, we indeed derive a solar abundance of $A(\mathrm{Ba})=2.21 \mathrm{dex}$ from this feature, in nice agreement with accepted values for solar $\mathrm{Ba}$ abundances (see Table 1). We adopted an isotopic solar mixture of $81 \%\left({ }^{134} \mathrm{Ba}+{ }^{136} \mathrm{Ba}+{ }^{138} \mathrm{Ba}\right)$ and $19 \%\left({ }^{135} \mathrm{Ba}+{ }^{137} \mathrm{Ba}\right)$, as in all our previous studies (e.g. Desidera et al. 2011; D'Orazi et al. 2012; De Silva et al. 2013). In the parameter range of our sample stars, departures from LTE for the Ba II lines under scrutiny here are very small, thus we did not include them (see e.g. Korotin et al. 2015).

\section{Error budget}

External (systematic) and internal (random) errors affect abundance analysis determinations. However, given the differential nature of our analysis, for the present purpose we can focus only on internal uncertainties, neglecting zero-point effects of our atmospheric parameters and elemental analysis. Two sources of errors can be disentangled:

(i) Errors related to the EW measurements (and/or spectral synthesis) that reflect uncertainties due to the continuum placement and the measurement procedure itself; they can be represented by the standard deviation from the mean (r.m.s), when more than one line for a species is available. For elements for which we are forced to rely only on one single feature $(\mathrm{K}, \mathrm{Zn})$, we repeated the measurement several times by changing the continuum displacement and other criteria and inspected the corresponding variation in the resulting abundances. Typical errors (labelled as observational errors in Table 4) range from 0.018 to 0.105 dex in $[\mathrm{X} / \mathrm{H}]$ ratios.

(ii) Errors due to atmospheric parameters. In order to assess the contribution related to the stellar parameters, we first need to ascertain the sensitivities of our species to changes in atmospheric quantities. To do this, we proceeded in the standard way, by varying one parameter at the time and inspecting the corresponding change in the resulting abundance. The following step is to evaluate the actual error in atmospheric parameters that originates from errors on the slope for $T_{\text {eff }}$ and $\xi$; when estimating the uncertainty in gravity we took into account both the standard error from EW measurements of the FeII and the error in $T_{\text {eff }}$; that is an error of $20 \mathrm{~K}$ would produce a variation in $\log g$ of $0.06 \mathrm{dex}$. We found values of $20 \mathrm{~K}, 0.07 \mathrm{dex}, 0.1 \mathrm{~km} \mathrm{~s}^{-1}$, and $0.04 \mathrm{dex}$ for temperature, gravity, microturbulence, and input metallicity, respectively. The different contributions are subsequently added in quadrature to gather the total uncertainties due to the stellar parameters (labelled as Param in Table 4).

Given the very (internally) accurate parameter determination, the observational scatter is by far the dominant source of error in our abundances.

\subsection{Results}

Our results for the two reference stars, ८ Hor and HIP 84827, provide the following atmospheric parameters and metallicity. We obtained $T_{\text {eff }}=6230 \pm 60 \mathrm{~K}, \log g=4.45 \pm 0.15 \mathrm{dex}$, $\xi=1.06 \pm 0.10 \mathrm{~km} \mathrm{~s}^{-1}$ and $[\mathrm{Fe} / \mathrm{H}]=0.19 \pm 0.06 \mathrm{dex}$ and $T_{\mathrm{eff}}=$ $6155 \pm 60 \mathrm{~K}, \log g=4.15 \pm 0.15 \mathrm{dex}, \xi=1.00 \pm 0.10 \mathrm{~km} \mathrm{~s}^{-1}$ and $[\mathrm{Fe} / \mathrm{H}]=0.19 \pm 0.08$ dex for $\iota$ Hor and HIP 84827 , respectively. We would like to emphasise that both estimates are in very good agreement with previous determinations of parameters and abundances for the two reference stars, suggesting that systematic uncertainties do not largely affect our analysis (see for example Biazzo et al. 2012; and Ramírez et al. 2014). More importantly, our parameter determination for the planethost star $\iota$ Hor agrees within $1 \sigma$ with the asteroseismic results (see Vauclair et al. 2008).

The differential (line-by-line) spectroscopic analysis provides the following differential parameters for GJ 504 when $\iota$ Hor is taken as reference: $\mathrm{d} T_{\text {eff }}=-40 \pm 20 \mathrm{~K}$, dlog $g=$ $-0.20 \pm 0.07 \mathrm{dex}, \mathrm{d} \xi=+0.15 \pm 0.10 \mathrm{~km} \mathrm{~s}^{-1}$ and $\mathrm{d}[\mathrm{Fe} / \mathrm{H}]=$ $+0.03 \pm 0.03$ dex. On the other hand, we found $\mathrm{d} T_{\text {eff }}=+65 \pm$ $20 \mathrm{~K}, \log g=+0.17 \pm 0.07 \mathrm{dex}, \mathrm{d} \xi=+0.24 \pm 0.10 \mathrm{~km} \mathrm{~s}^{-1}$ and $\mathrm{d}[\mathrm{Fe} / \mathrm{H}]=+0.03 \pm 0.04$ dex when the analysis is differential with respect to HIP 84827 . We then calculated the absolute abundances for GJ 504 relative to these reference stars and we finally took the average values as our adopted stellar parameters and metallicity, that is $T_{\text {eff }}=6205 \pm 20 \mathrm{~K}, \log g=4.29 \pm 0.07$ dex, $\xi=1.23 \pm 0.10 \mathrm{~km} \mathrm{~s}^{-1}$ and $[\mathrm{Fe} / \mathrm{H}]=0.22 \pm 0.04$ dex. In Table 2 we list for GJ 504, along with our stellar parameters and $[\mathrm{Fe} / \mathrm{H}]$, literature values from several recent studies, based on high-resolution spectroscopy. The temperature is in good agreement with previous estimates as well as metallicity and microturbulence values; we confirm that the star is characterised by a super-solar metallicity. The crucial result here is represented by the surface gravity. Our analysis (as the vast majority of previous spectroscopic studies with the exception of Valenti \& Fischer 2005; and Maldonado et al. 2012) indicates that the star is actually slightly evolved, and compatible with the turn-off evolutionary stage. We stress that the agreement between the temperature value from the present work and Valenti \& Fischer (2005), but a strong mismatch in gravity, is not surprising because in their paper gravities have been evaluated from the fitting of the $\mathrm{Mg} \mathrm{b}$ lines; hence they are uncorrelated with $T_{\text {eff }}$. Thus our result is in contrast to what has been suggested by Kuzuhara et al. (2013), who adopted gravity from Valenti \& Fischer (2005) (i.e. young age), corroborating the conclusions of Fuhrmann and Chini. The implications of our results in terms of age are discussed in the next Section and are crucial when interpreting the substellar nature of the companion (see also the introduction of this paper).

At first glance, other element abundances track the iron content reasonably well. A closer inspection reveals, however, that GJ 504 exhibits a small overabundance in $\mathrm{K}$, which is $[\mathrm{K} / \mathrm{Fe}]=$ $0.15 \pm 0.08$ dex. When observational errors are taken into account this value is still compatible with a solar-scaled pattern. Moreover, carbon and oxygen are underabundant. We recall that since NLTE corrections have been applied, our results do not depend on the wrong assumption of local thermodynamical equilibrium. Had not we applied this corrections, the $[\mathrm{K} / \mathrm{Fe}]$ ratios would have been $\approx 0.50 \mathrm{dex}$. The same holds for oxygen abundances, for which NLTE corrections are about -0.3 dex. It is noteworthy in this context, that Bensby et al. (2014) obtained a $[\mathrm{O} / \mathrm{H}]=0.11 \pm 0.11 \mathrm{dex}$ (thus subsolar $[\mathrm{O} / \mathrm{Fe}]$ ratio), which is in agreement with our value once errors are considered. Besides, the impact of NLTE departures is actually much smaller when we consider differential abundances of GJ 504 with respect to $\iota$ Hor because the stars are extremely similar in terms of atmospheric parameters with the clear exception of gravity (where $\Delta \log g=0.20 \pm 0.07 \mathrm{dex}$ ).

The $\mathrm{Li}$ abundance is discussed in the next section, which is dedicated to a reassessment of all the other age indicators. The Li content is indeed known to be a critical key diagnostics for 
Table 2. Comparison between GJ 504 stellar parameters and metallicity from this work and several, recent literature values.

\begin{tabular}{lcccr}
\hline \hline $\begin{array}{l}T_{\text {eff }} \\
(\mathrm{K})\end{array}$ & $\begin{array}{c}\log g \\
(\mathrm{dex})\end{array}$ & $\begin{array}{c}\xi \\
\left(\mathrm{km} \mathrm{s}^{-1}\right)\end{array}$ & $\begin{array}{c}{[\mathrm{Fe} / \mathrm{H}]} \\
(\mathrm{dex})\end{array}$ & Reference \\
\hline $6205 \pm 20$ & $4.29 \pm 0.07$ & $1.23 \pm 0.10$ & $0.22 \pm 0.04$ & This work \\
$5978 \pm 60$ & $4.23 \pm 0.10$ & $1.13 \pm 0.20$ & $0.13 \pm 0.06$ & Fuhrmann \& Chini (2015) \\
$6234 \pm 25$ & $4.60 \pm 0.02$ & - & $0.28 \pm 0.03$ & Valenti \& Fischer (2005) \\
$6012 \pm 100$ & $4.30 \pm 0.20$ & $1.10 \pm 0.20$ & $0.11 \pm 0.10$ & Mishenina et al. (2013) \\
$6185 \pm 51$ & $4.30 \pm 0.07$ & $1.28 \pm 0.08$ & $0.25 \pm 0.06$ & Battistini \& Bensby (2015) \\
$5995 \pm 41$ & $4.24 \pm 0.02$ & $1.34 \pm 0.12$ & $0.11 \pm 0.04$ & Ramírez et al. (2013) \\
$6133 \pm 50$ & $4.63 \pm 0.13$ & $1.30 \pm 0.26$ & $0.24 \pm 0.04$ & Maldonado et al. (2012) \\
\hline
\end{tabular}

Table 3. Differential abundances of GJ 504 with respect to $\iota$ Hor and HIP 84827 in Cols. (2) and (3), respectively.

\begin{tabular}{lcccc}
\hline \hline Elem. & $\begin{array}{c}\mathrm{d}[\mathrm{X} / \mathrm{H}] \\
(\text { w.r. } \iota \text { Hor })\end{array}$ & Obs. & $\begin{array}{c}\mathrm{d}[\mathrm{X} / \mathrm{H}] \\
\text { (w.r.t HIP } 84827)\end{array}$ & Obs. \\
\hline $\mathrm{C}$ & -0.057 & 0.083 & 0.000 & 0.082 \\
$\mathrm{O}$ & -0.031 & 0.063 & 0.050 & 0.030 \\
$\mathrm{Na}$ & 0.019 & 0.110 & -0.066 & 0.101 \\
$\mathrm{Mg}$ & 0.030 & 0.027 & -0.083 & 0.087 \\
$\mathrm{Al}$ & 0.023 & 0.082 & 0.120 & 0.031 \\
$\mathrm{Si}$ & 0.067 & 0.058 & 0.008 & 0.065 \\
$\mathrm{~S}$ & -0.099 & 0.077 & -0.023 & 0.076 \\
$\mathrm{~K}$ & -0.010 & 0.070 & 0.120 & 0.070 \\
$\mathrm{Ca}$ & 0.046 & 0.056 & 0.080 & 0.047 \\
$\mathrm{Ti}$ & 0.019 & 0.016 & 0.015 & 0.053 \\
$\mathrm{Cr}$ & -0.028 & 0.021 & -0.020 & 0.048 \\
$\mathrm{Fe}$ & 0.009 & 0.026 & 0.011 & 0.065 \\
$\mathrm{Ni}$ & 0.060 & 0.065 & -0.029 & 0.063 \\
$\mathrm{Zn}$ & 0.035 & 0.028 & -0.073 & 0.045 \\
\hline
\end{tabular}

Notes. Oxygen has been corrected for 3D+NLTE effects, following prescriptions by Amarsi et al. (2015). Similarly we provide NLTE K abundances. Listed values for $\mathrm{Ti}, \mathrm{Cr}$, and $\mathrm{Fe}$ are the average values between singly ionised and neutral lines.

stellar ages of relatively cool stars, as shown by numerous studies (e.g. Sestito \& Randich 2005). Here we mention that we obtained $A(\mathrm{Li})=2.88 \pm 0.07$, from the spectral synthesis of the $\mathrm{Li}$ doublet. This value agrees very well with previous analyses by Takeda \& Kawanomoto (2005) and Ramírez et al. (2013), who obtained $A(\mathrm{Li})=2.91 \pm 0.10$ and $A(\mathrm{Li})=2.85 \pm 0.04$, respectively.

Finally, the barium abundance deserves a special, dedicated discussion. In 2009, we discovered that there is a negative correlation between the $\mathrm{Ba}$ content and the open cluster age (D'Orazi et al. 2009). This finding was confirmed by other authors (e.g. Jacobson \& Friel 2013), providing further evidence that the younger the cluster, the higher the $\mathrm{Ba}$ abundance. Premain-sequence clusters, such as IC 2602 and IC 2391 (age between 30 and $50 \mathrm{Myr}$ ), exhibit $[\mathrm{Ba} / \mathrm{Fe}]$ ratios up to $\approx 0.65 \mathrm{dex}$, that is more than a factor of four the solar value. Since then, a variety of studies have been conducted to ascertain the nature of this peculiar trend, which does not seem to be accompanied by a similar behaviour from the other elements produced in slow neutron-capture reactions (La in particular, which belongs with $\mathrm{Ba}$ to the second peak of the $s$-process path). Regardless the nature of such a special chemical pattern (see an extensive dis- cussion in D'Orazi et al. 2012), we can exploit this overabundance as a youth indicator. Crucial to the present work, supersolar Ba ratios in young stars are not confined to the cluster environment, but it has also been shown to be present also in young, isolated stars (see e.g. HD 61005 by Desidera et al. 2011). Our analysis indicates that GJ504 is characterised by a solar $\mathrm{Ba}$ abundance, which is $[\mathrm{Ba} / \mathrm{Fe}]=-0.04 \pm 0.01 \pm 0.03$ dex; the second error is from stellar parameters, whereas the first is simply the standard deviation from the two different $\mathrm{Ba}$ features. On a differential scale with respect to $\iota$ Hor, GJ 504 has $\Delta[\mathrm{Ba} / \mathrm{Fe}]=-0.28 \pm 0.05$ dex, implying an enhanced $\mathrm{Ba}$ content for the reference star, at variance with the allegedly young age of GJ 504. Although based on a different analysis procedure, we confirm that $\iota$ Hor is rich in $\mathrm{Ba}$, as previously determined in D'Orazi et al. (2012) and in nice agreement with expectations, according to the young age. This is a very important result because the age and the evolutionary stage of $\iota$ Hor are very well constrained, given the asteroseismic measurements published by Vauclair et al. (2008). Thus, we can conclude that our analysis for $\mathrm{Ba}$ abundance determination (along with the gravity) also points to an old age for GJ 504 .

\section{Other age indicators}

\section{Rotational period, chromospheric activity and X-ray emission}

The rotation period $P=3.33 \mathrm{~d}$ is well established from longterm Ca II H\&K time series (Donahue et al. 1996) and photometry (Messina 1998). Donahue et al. also detected rotation period variations in the range from $3.23 \mathrm{~d}$ to $3.41 \mathrm{~d}$ that they attributed to the presence of surface differential rotation.

This rotation period of $3.33 \mathrm{~d}$ is intermediate between those of stars of similar colours of the Hyades and Pleiades open clusters (Fig. 3). The stellar radius as derived in Sect. 3 allows us to estimate the inclination at which the star is seen by the Earth. From the rotation period of 3.33 days and the projected rotational velocity $v \sin i=6 \pm 1 \mathrm{~km} \mathrm{~s}^{-1}$ (calculated as a by-product of the spectral synthesis method; see also e.g. Gonzalez et al. 2010), we infer a system inclination of $18 \mathrm{deg}$. The nearly poleon inclination does not prevent the detectability of photometric rotational modulations, as discussed in Messina et al. (2016). It is possible that the true rotation period is two times the proposed one, with the shorter periodicity resulting from two roughly symmetric active latitudes. As the original datasets are not available, we cannot conclusively support or discard this possibility, but we emphasise that a period of 6.6 days would not solve the discrepancy with ages coming from isochrones and spectroscopy. Thus our conclusions are unaffected.

The average index $\log R_{\mathrm{HK}}^{\prime}=-4.446$ (Baliunas et al. 1996) is on the higher envelope of Hyades members of same mass 
Table 4. $[\mathrm{X} / \mathrm{H}]$ ratios for GJ 504 with respect to the Sun and errors (see text).

\begin{tabular}{lcccccccc}
\hline \hline Element & {$[\mathrm{X} / \mathrm{H}]$} & $\begin{array}{c}\Delta T_{\text {eff }} \\
(+20 \mathrm{~K})\end{array}$ & $\begin{array}{c}\Delta \log g \\
(+0.07 \mathrm{dex})\end{array}$ & $\begin{array}{c}\Delta \xi \\
\left(+0.10 \mathrm{~km} \mathrm{~s}^{-1}\right)\end{array}$ & $\begin{array}{c}\Delta[\mathrm{M} / \mathrm{H}] \\
(+0.04 \mathrm{dex})\end{array}$ & Param & Observational & Total \\
\hline $\mathrm{C}$ & -0.004 & 0.008 & -0.027 & -0.005 & -0.008 & 0.026 & 0.106 & 0.109 \\
$\mathrm{O}$ & 0.030 & 0.024 & -0.010 & 0.029 & 0.015 & 0.043 & 0.040 & 0.059 \\
$\mathrm{Na}$ & 0.156 & 0.154 & 0.158 & 0.156 & 0.158 & 0.003 & 0.036 & 0.036 \\
$\mathrm{Mg}$ & 0.201 & 0.197 & 0.211 & 0.199 & 0.201 & 0.011 & 0.060 & 0.061 \\
$\mathrm{Al}$ & 0.187 & 0.184 & 0.193 & 0.185 & 0.187 & 0.007 & 0.018 & 0.019 \\
$\mathrm{Si}$ & 0.215 & 0.216 & 0.212 & 0.213 & 0.213 & 0.004 & 0.090 & 0.090 \\
$\mathrm{~S}$ & 0.000 & -0.003 & -0.021 & -0.002 & -0.003 & 0.022 & 0.024 & 0.033 \\
$\mathrm{~K}$ & 0.370 & 0.010 & -0.010 & -0.030 & 0.000 & 0.030 & 0.070 & 0.080 \\
$\mathrm{Ca}$ & 0.268 & 0.264 & 0.283 & 0.263 & 0.267 & 0.016 & 0.105 & 0.106 \\
$\mathrm{Ti}$ & 0.192 & 0.189 & 0.173 & 0.190 & 0.191 & 0.019 & 0.027 & 0.033 \\
$\mathrm{Cr}$ & 0.194 & 0.192 & 0.176 & 0.190 & 0.193 & 0.019 & 0.033 & 0.038 \\
$\mathrm{Fe}$ & 0.210 & 0.222 & 0.213 & 0.199 & 0.225 & 0.022 & 0.055 & 0.059 \\
$\mathrm{Ni}$ & 0.233 & 0.232 & 0.234 & 0.231 & 0.231 & 0.003 & 0.056 & 0.056 \\
$\mathrm{Zn}$ & 0.209 & 0.210 & 0.209 & 0.195 & 0.203 & 0.015 & 0.050 & 0.052 \\
\hline
\end{tabular}

(Fig. 3). The $\log R_{\mathrm{HK}}^{\prime}$ given in Baliunas et al. (1996) is slightly different $(-4.443)$ because of the slightly different $B-V$ colour they adopted. We rederived the value of $\log R_{\mathrm{HK}}^{\prime}$ for our adopted colour $B-V=0.585$ for consistency with the other indicators. The Baliunas et al. (1996) $\log R_{\mathrm{HK}}^{\prime}$ is based on a very extended time series of several hundreds of measurements spanning 20 years, then effectively averaging variability on daily and yearly timescales. Several other literature sources quote $\log R_{\mathrm{HK}}^{\prime}$ for GJ 504, mostly based on sparse measurements. They are consistent with the Baliunas et al. (1996) results. The coronal activity, as measured by ROSAT, is also consistent with the fast rotation and high activity level of the star (Fig. 3). A photometric time series that is $13 \mathrm{yr}$ long and an HK time series that is $20 \mathrm{yr}$ long show that the photometric and chromospheric variability are found to be anti-correlated with a significance level $>95 \%$. This behaviour is typical of young active stars (see e.g. Lockwood et al. 2007, their Fig. 3). The estimated stellar ages derived from rotation, $\log R_{\mathrm{HK}}^{\prime}$, and $\log L_{X} / L_{\mathrm{bol}}$ are $163 \mathrm{Myr}, 440 \mathrm{Myr}$, and $431 \mathrm{Myr}$, respectively, when using Mamajek \& Hillenbrand (2008) calibrations. In summary, the age indicators linked to stellar rotation indicate an age intermediate between the Pleiades and the Hyades. The rotational and activity parameters of $\iota$ Hor are $P_{\text {rot }}=8.5 \mathrm{~d}$; $\log R_{\mathrm{HK}}^{\prime}=-4.61$ and $\log L_{\mathrm{X}} / L_{\mathrm{bol}}=-4.97$ (Metcalfe et al. 2010). Therefore, $\iota$ Hor results to be slightly older than GJ 504 from the rotation/activity point of view and with a high accuracy age of $625 \pm 5 \mathrm{Myr}$ from asteroseismology (Vauclair et al. 2008), further supporting our choice as reference young star in the differential abundance analysis.

\section{Lithium abundance}

The lithium content of the stellar atmosphere is another key age diagnostic, although it is not as sensitive as for cooler stars for effective temperature as that of GJ 504. To avoid the challenges due to possible systematic differences in temperature scale we compared the equivalent width of Li line at $6707 \AA$ of GJ 504 (and $\iota$ Hor) with those of members of open clusters of different ages (Pleiades, Hyades, and NGC 752). Lithium data were taken from Soderblom et al. (1993), Thorburn et al. (1993), and
Sestito et al. (2004) for Pleiades, Hyades, and NGC 752, respectively. As shown in Fig. 3, the Li EW of GJ 504 lies on the locus of Hyades members, then with a marginal discrepancy with respect to the younger age inferred from rotation, and clearly above the locus of the 2 Gyr-old open cluster NGC 752. GJ 504 has a temperature that puts it on the cool border of the Li dip. Therefore, its Li content could be slightly larger than observed and in better agreement with an age younger than the Hyades.

\section{Kinematic parameters}

GJ 504 is not associated with any known young moving group. The heliocentric space velocity components $\mathrm{U}, \mathrm{V}, \mathrm{W}$ are found to be $-38.0 \pm 0.2,+1.3 \pm 0.2$, and $-17.8 \pm 0.5 \mathrm{~km} \mathrm{~s}^{-1}$, respectively, when adopting the proper motion and trigonometric parallax from van Leeuwen (2007) and the absolute radial velocity (RV = $-27 \mathrm{~km} \mathrm{~s}^{-1}$ ) from Nidever et al. (2002). These parameters are slightly outside the kinematic space of young stars identified by Montes et al. (2001), making an age as young as 200-300 Myr unlikely. The BANYAN II on-line tool (Gagné et al. 2014) also yields a much higher probability that the star belongs to the old field rather than the young field (97\% versus $3 \%)$. The galactic orbit derived by Marsakov \& Shevelev (1995) $\left(R_{p}=7.98 \mathrm{kpc}\right.$; $R_{a}=10.00 \mathrm{kpc} ; Z_{\max }=0.128 \mathrm{kpc}$, and $e=0.112$ ) further rules out a very young age but it is still in fair agreement with an age that is a bit younger than the Sun, as argued by the isochrone fitting in Sect. 3.

\section{Isochrones}

We derived the stellar ages from the isochrone fitting using both the observed colours and magnitudes and the spectroscopic parameters from Sect. 2.2. We used the PARSEC models by Bressan et al. (2012) for the appropriate metallicity of the star $(Z=0.0235 \text {, corresponding to }[\mathrm{M} / \mathrm{H}]=+0.22)^{4}$, adopting $\mathrm{Z}_{\odot}=$ 0.0152 as for PARSEC isochrones; see Bressan et al. (2012).

\footnotetext{
4 http://stev.oapd.inaf.it/cgi-bin/cmd, PARSEC ver-
} sion $1.2 \mathrm{~S}$. 

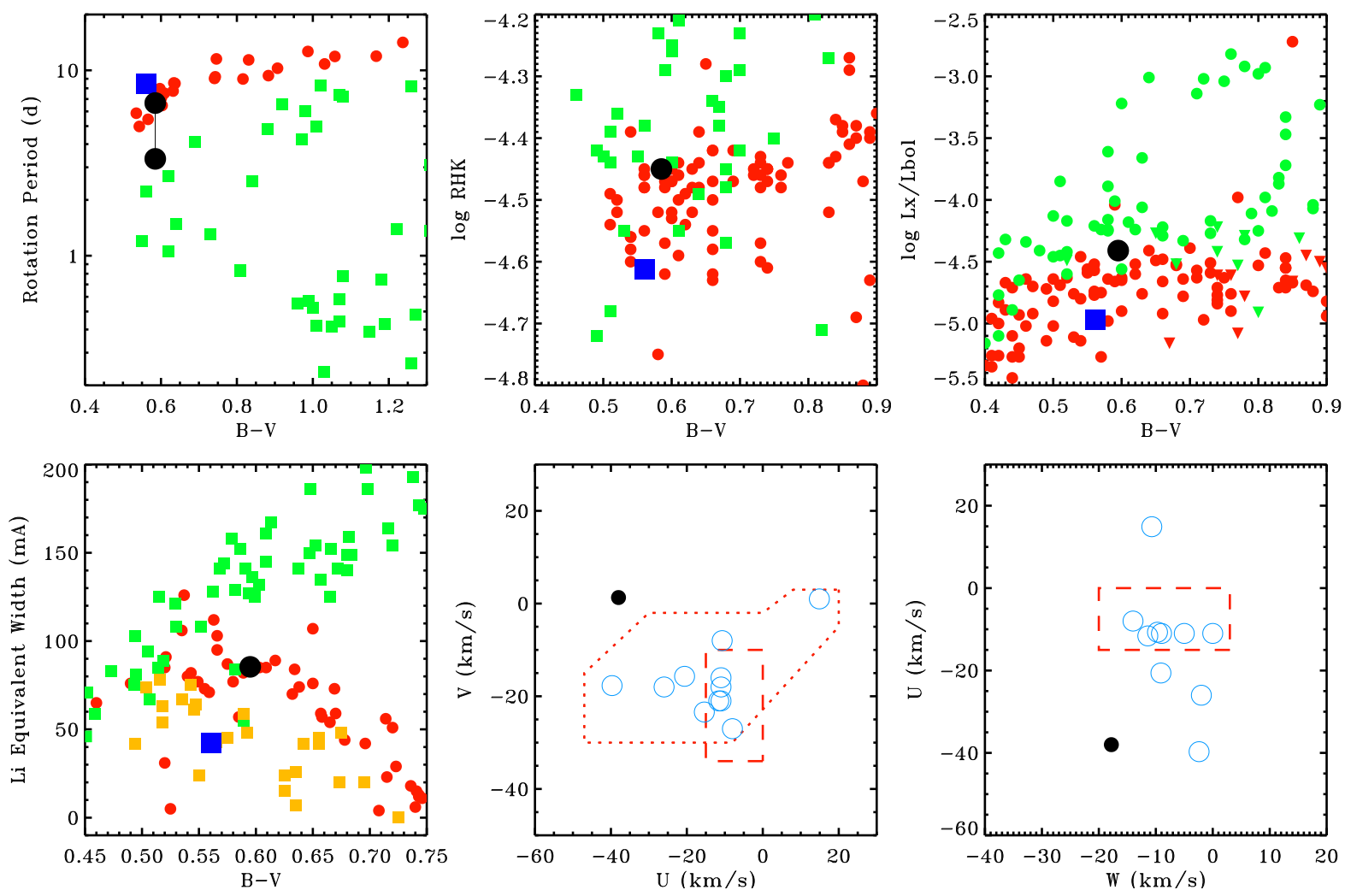

Fig. 3. Age indicators for GJ 504 compared to those of $\iota$ Hor and open clusters of well-determined age. In the various panels, GJ 504 is shown as a filled black circle, $\iota$ Hor as a filled blue square, members of the Pleiades open cluster as green filled squares, members of the Hyades open cluster as red circles, and members of the NGC 752 open cluster as orange filled squares. Upper left panel: rotation period vs. $B-V$. For GJ 504, both the literature rotation period $(3.33 \mathrm{~d})$ and the possible alternative period that is two times longer are plotted and connected by a solid line. Upper middle panel: $\log R_{\mathrm{HK}}^{\prime}$ Vs. $B-V$. Upper right panel: $\log L_{\mathrm{X}} / L_{\mathrm{bol}}$ Vs. $B-V$. In these plots, red filled triangles and green filled triangles represent the stars with X-ray upper limits members of Hyades and Pleiades, respectively. Lower left panel: lithium $E W$ vs. $B-V$ for GJ 504 (black square). Lower middle panel: $U$ vs. $V$ space velocities for GJ 504 and young moving groups (open circles). Overplotted are the boundaries of the kinematic space populated by young stars. Lower right panel: $W$ vs. $U$ space velocities for GJ 504 and young moving groups (open circles).

Figure 4 shows the colour magnitude diagrams using $V$ magnitude and $B-V$ and $V-I$ colours. GJ 504 lies about $0.3 \mathrm{mag}$ above the ZAMS, close to the 3.0 and $3.2 \mathrm{Gyr}$ isochrones, respectively. When using the $T_{\text {eff }}$ versus $M_{V}$ diagram, GJ 504 appears marginally younger (age about $2.1 \mathrm{Gyr}$ ). The age as resulting from the $\log g$ versus $T_{\text {eff }}$ diagram is $3.0 \mathrm{Gyr}$.

If the position above main sequence is instead interpreted as a pre-main-sequence evolutionary phase, the resulting isochrone age is of about 20-25 Myr. Such an extremely young age is definitely not consistent with the observed lithium content and the kinematic parameters (space velocities very far from the typical locus of stars younger than $100 \mathrm{Myr}$ ).

We then conclude that the isochrone age of GJ 504 is between 1.8 and $3.5 \mathrm{Gyr}$ with a most probable value of $2.5 \mathrm{Gyr}$. The corresponding stellar radii and masses are $1.30 R_{\odot}$ and $1.22 M_{\odot}$, respectively. We stress that the absolute age determination of GJ 504 is beyond the scope of the present paper and this will be addressed in a forthcoming work that will focus on asteroseismic observations of the system. For our purposes only differential properties of GJ 504 with respect to the reference stars (especially $\iota$ Hor) are relevant.

Our estimate of the isochronal age indicates that GJ 504 is slightly younger than that derived by Fuhrmann \& Chini (2015). This is mostly because of the different values of effective temperature adopted. However, the discrepancy of isochrone age with other age indicators such as rotation, activity and lithium is maintained and calls for a dedicated explanation. The isochrone age is instead consistent with the kinematic parameters and the barium abundance.

\section{Age summary}

We explored whether the isochrone age, as resulting from the evolved position in the colour-magnitude diagram, could be spurious as a result of an unresolved binarity of GJ 504 .

There are no indications of additional stellar components contributing to the integrated flux either from direct imaging (ruling out equal-luminosity companions down to a separation of 30-50 mas; Bonnefoy et al., in prep.), or from spectroscopy. Radial velocity (RV) data from Fischer et al. (2014), direct inspection of the FEROS spectra, and the check for possible dependencies of the results of the abundance analysis on wavelength of the spectral lines seems to exclude this scenario. Only very special orbit configurations (wide orbits with extremely small projected separation at the time of the imaging observations) are not yet ruled out by available observations, however their occurrence probability is vanishingly small because of tight observational constraints. In any case a blended binary scenario would not easily explain the fully consistent result between the spectroscopic gravity and the position on colour-magnitude diagram. The isochrone age is also supported by the kinematic parameters and barium abundance and any ad-hoc explanation for a spurious estimate would not remove the discrepancy for these indicators. Therefore, we dismiss the possibility that the isochrone age is biased by an unresolved companion and look for alternative 

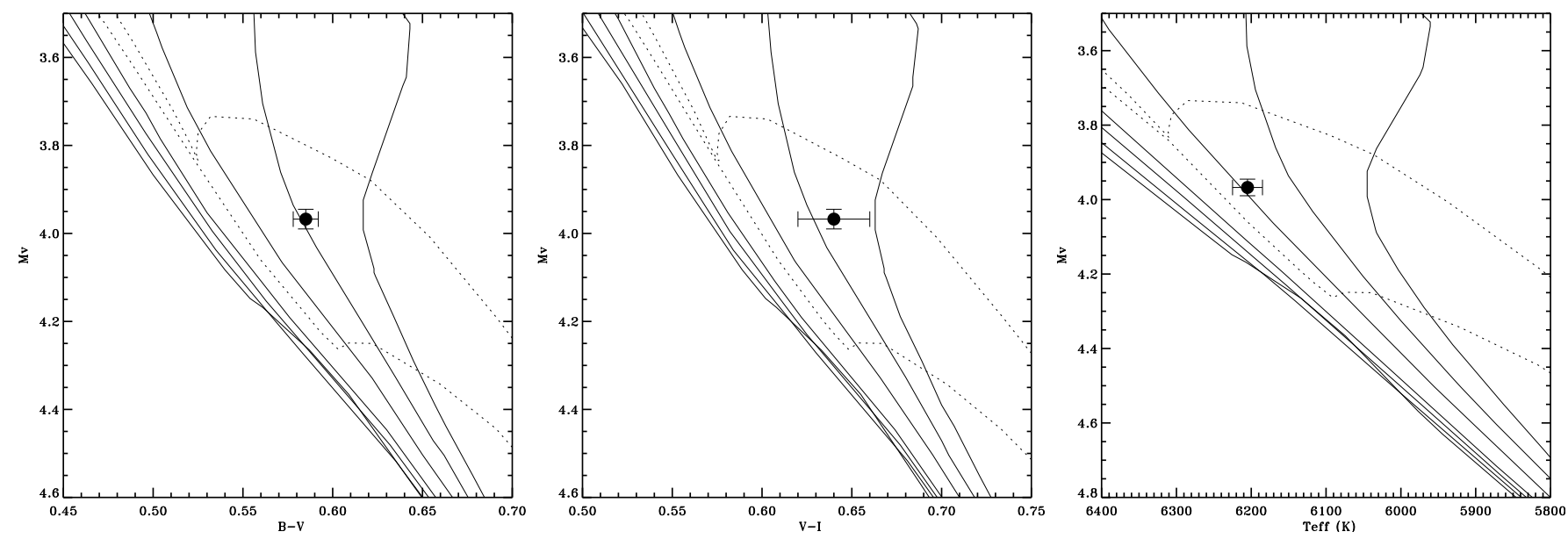

Fig. 4. $M_{V}$ vs. $B-V$ (left panel), $M_{V}$ vs. $V-I$ (central panel), and $M_{V}$ vs. $T_{\text {eff }}$ (right panel) for GJ 504. Overplotted are the 0.1, 0.4, 0.7, 1.0, 2.0, 3.0, and 4.0 Gyr isochrones (continuous lines) and the 20 and $25 \mathrm{Myr}$ pre-main-sequence isochrones (dashed lines) for the appropriate metallicity, from Bressan et al. (2012).

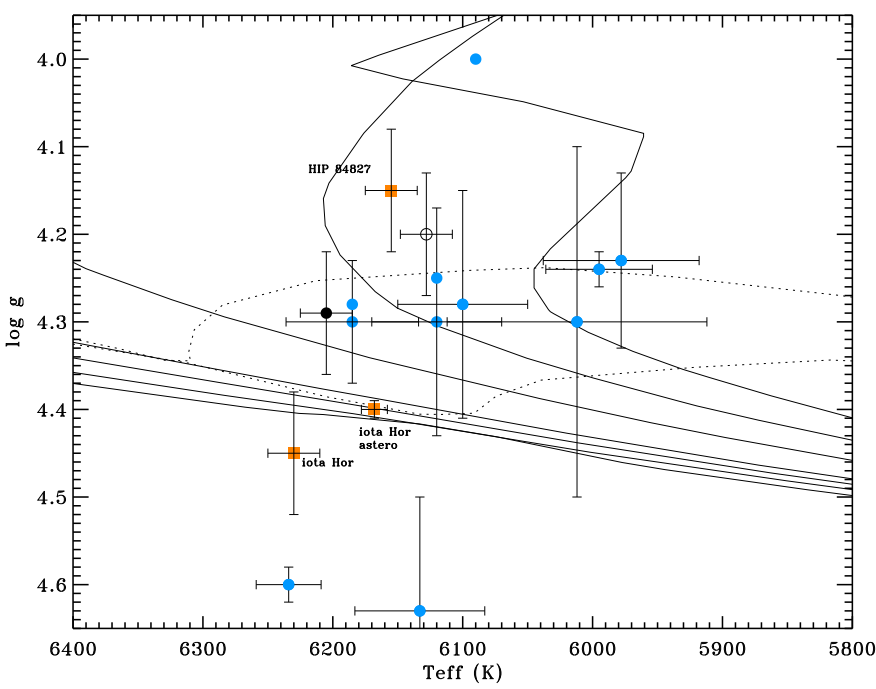

Fig. 5. $\log g$ vs. $T_{\text {eff }}$ for GJ 504 as resulting from our spectroscopic analysis (filled black circle) and when applying the results of the spectroscopic differential analysis with respect to the asteroseismology value of $\iota$ Hor (empty circle). Overplotted are the 0.1, 0.4, 0.7, 1.0, 2.0, 3.0, and $4.0 \mathrm{Gyr}$ isochrones (continuous lines) and the 20 and $25 \mathrm{Myr}$ premain-sequence isochrones (dashed lines) for the appropriate metallicity from Bressan et al. (2012). Additional measurements of $\log g$ vs. $T_{\text {eff }}$ for GJ 504 from the literature are plotted as light blue filled circles. The parameters of $\iota$ Hor and HIP 84827 (our spectroscopic analysis and the parameters of iota Hor) as derived from the asteroseismology analysis (Vauclair et al. 2008) are plotted as empty red circles.

explanations for the discrepancy with the other indicators (rotation, activity, and lithium), assuming that the isochrone age is the most reliable for GJ 504.

\section{Solving the age discrepancy: merging events}

\subsection{Ruling out stellar companions and their remnants}

The discrepancy between the age indicators linked to rotation and the placement on HR diagram can be ascribed to the interaction with a companion, which could spin up the central star. This could happen through tidal interactions by a close companion, a merging, or wind accretion by a moderately wide companion at the end of the AGB phase.
The presence of a massive companion close enough to tidally spin up the central star is ruled out by the RV monitoring performed at Lick Observatory (Fischer et al. 2014). The RV time series include 58 measurements over 21.65 years. The observed $\mathrm{RV}$ dispersion $\left(25.7 \mathrm{~m} \mathrm{~s}^{-1}\right)$ is fairly large but comparable with the high activity level of the star. This allows us to exclude the presence of close companions more massive than about $0.5-1 M_{J}$.

The lack of any long-term RV slope also argues against the presence of a white dwarf (WD) companion at a separation small enough to have allowed significant accretion of material and angular momentum onto the central star (see Zurlo et al. 2013, for details and references on this scenario). Such a white dwarf companion is also ruled out by the imaging observations. Finally, a hot WD would be expected if a recent accretion event had occurred (as expected from the fast rotation of GJ 504), while the far and near ultra violet magnitudes observed by GALEX are fully consistent with those of a chromospherically active G0V star (Findeisen et al. 2011) and clearly rule out a spatially unresolved hot WD companion to GJ 504.

This leaves the occurrence of a merging event as the most viable possibility to account for the various observational constraints, as already proposed by Fuhrmann \& Chini (2015). Following Fuhrmann \& Chini (2015), it should be noticed that the high lithium content allows us to constrain the mass of the merged object. Indeed, the merging with a low-mass star of $0.1-0.2 M_{\odot}$ would imply that the material accreted is completely depleted in lithium and an original mass of 1.0-1.1 $M_{\odot}$ for the primary would imply some Li depletion after 2-3 Gyr. The observed Li EW of GJ 504 is instead above the locus of the 1.5-2.0 Gyr old NGC 752 open cluster. This is not consistent with the original $\mathrm{Li}$ abundance of a star with mass significantly below the present one and the accretion of a significant amount of fully $\mathrm{Li}$ depleted material. This suggests that the original mass of the primary was very close to the present one, and that the mass of the merged companion was substellar and most likely low enough to have preserved lithium. From the observed mass distribution of close-in substellar companions (Reggiani et al. 2016, and references therein), it is more likely to face a planet rather than a brown dwarf. We briefly mention, in this context, that the possible difference in lithium content for stars with and without planets is a very debated topic and no final conclusion has been drawn yet. Delgado Mena et al. (2014) and 
references therein, for example provide for a dedicated discussion; however, the temperature of GJ 504 is hotter than the range $\left(T_{\text {eff }} \approx 5600-5900 \mathrm{~K}\right)$, where evidence for a lower Li abundance in planet-host stars has been observed. Moreover, it is clear that GJ 504 belongs to a completely different class of objects because the central star has already cannibalised the close-in giant planet and we do not know what kind of planetary system was present at the beginning of its evolution. On the other hand, all correlations between $\mathrm{Li}$ and planet hosts are focussed on stars currently orbited by planetary-mass companions, hence engulfment events did not occur.

We discuss the scenario of the engulfment of a planetary companion in more detail in the next subsection.

\subsection{A possible engulfment scenario of a planetary companion}

To quantitatively evaluate the timescales involved in our proposed engulfment scenario, we employed a simple tidal evolution model. It assumes circular and coplanar orbits and includes the rate of angular momentum loss produced by the stellar magnetised wind $\dot{L}_{\mathrm{w}}$ with a Skumanich-type law; that is $\dot{L}_{\mathrm{w}}=-K \Omega^{3}$, where $K=3.52 \times 10^{40} \mathrm{~kg} \mathrm{~m}^{2} \mathrm{~s}$ and $\Omega$ is the stellar angular velocity. The tidal torque between the star and the planet follows the equilibrium-tide formalism (see e.g. Zahn 2008) with its strength parameterised by the tidal modified quality factor $Q^{\prime}$ of the star. We take into account the evolution of the radius of the star along the main sequence ${ }^{5}$. The adopted parameters for stellar mass and metallicity are $M=1.2 M_{\odot}$ and $Z=0.02$, respectively. The validity of the Skumanich law implies that we cannot model phases with the star rotating faster than $\approx 5$ days. Following Fuhrmann \& Chini (2015), we adopt a mass of $2.7 M_{\mathrm{j}}$ for the planet.

Since the planet is falling into the star, the numerical solution of our tidal equations is highly sensitive to the initial conditions when we integrate them forwards in time. To avoid such an instability, we start from the final condition, that is with the planet filling its Roche lobe at $a_{\mathrm{f}}=0.009 \mathrm{AU}$, and integrate backwards in time to find the initial condition. Our model does not take into account the mass transfer that occurs when the planet fills its Roche lobe. This phase has been modelled by, for example Valsecchi et al. (2014, 2015) and Jackson et al. (2016), who found that its duration depends in a critical way on the interplay between the planet mass loss, which tends to increase the semi-major axis, and the stellar tides, which shrink it. We also note the above-mentioned publications concerning evaporation issues. A complete treatment of this topic is beyond the scope of the present paper and does not affect our conclusions. If the planet reaches its Roche lobe early, when the star is on the main sequence and tides are not particularly effective, the mass transfer phase can last for hundreds of Myr or Gyr. In our case, we assume that the planet loses most of its mass in a relatively short time (tens or hundreds Myr) because tides are rapidly shrinking its orbit owing to the radius expansion of our $1.2 M_{\odot}$ star during the final phase of its main-sequence evolution. Therefore, only the core of the planet, if any, should survive the rapid evolution after the onset of Roche lobe overflow.

We consider that the age of the star is $\approx 2$ Gyr and that the bulk of the mass transfer has not occurred more than $200 \mathrm{Myr}$ ago, otherwise the acquired angular momentum would have been

\footnotetext{
5 We have used the EZWeb interface to compute and read a simple stellar evolution model (http://www.astro.wisc.edu/ townsend/ static.php?ref=ez-web).
}
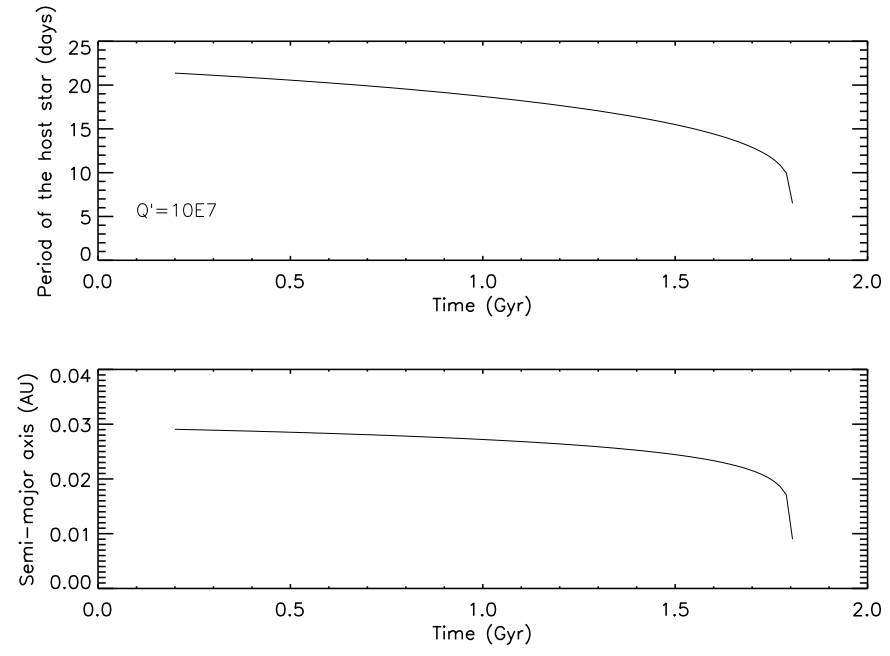

Fig. 6. Time evolution of the rotational period of the star (upper panel) and of the semi-major axis of the orbit of the engulfed planet (lower panel) for $Q^{\prime}=10^{7}$.
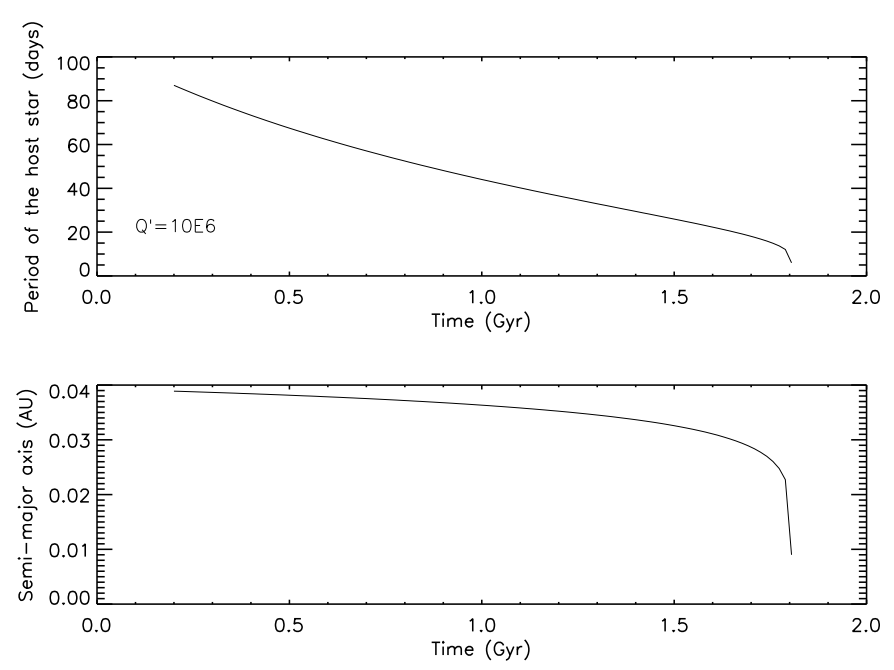

Fig. 7. As Fig. 6, but for $Q^{\prime}=10^{6}$.

partially lost resulting in a slowdown of the stellar rotation owing to its magnetised wind. For $Q^{\prime}=10^{7}$ and a stellar rotation period of 6.5 days when the planet reaches its Roche lobe, the reverse integration results in an initial orbital distance for the planet of $\approx 0.030 \mathrm{AU}$ and a rotation period of $\approx 20$ days (cf. Fig. 6). On the other hand, if we adopt $Q^{\prime}=10^{6}$, the system evolution is so fast and tides are so effective that the initial rotation period of the star becomes unrealistically long before $\approx 1$ Gyr (cf. Fig. 7). This is an indication that, despite all the uncertainties involved in our knowledge of the tidal interaction quality factor, $Q^{\prime} \approx 10^{7}$ seems more realistic. In comparison to the estimates by Fuhrmann \& Chini (2015), we find that the approach of the planet to the star under the action of tides is accompanied by a significant spin up of the star itself as a result of the transfer of angular momentum from the orbit to the stellar spin. Therefore, in our model the initial rotation of the star is found to be rather slow, if tides acted since the system had $\approx 0.2$ Gyr. We might speculate that the planet was not initially at $\approx 0.030 \mathrm{AU}$; but it was brought there through the Kozai mechanism because of the presence of the external massive planet/ brown dwarf companion of GJ504. Once this configuration was reached tidal forces did their job, causing an orbital decay in approximately 
1.8-2.0 Gyr. When the planet reached its Roche lobe, conservative mass transfer could have further decreased the rotation period of the star from $\approx 7$ days down to a few days. We can account for a faster initial stellar rotation at 0.2 Gyr by reducing the mass of the planet, its initial distance, and playing with the poorly known tidal parameter $Q^{\prime}$. In view of these uncertainties, we regard our computed models as simply illustrative and do not attempt a full exploration of the initial parameter space to account for the currently fast stellar rotation.

It is interesting to note that, according to our scenario in which the planet is initially based at a distance of $0.03 \mathrm{AU}$ with a mass of $2.7 M_{\mathrm{j}}$, the location of this hot Jupiter falls within the upper envelope of the distributions of planetary masses versus orbital period.

\section{Concluding remarks}

In this paper we have presented a comprehensive scrutiny of the fundamental properties for GJ 504. The star hosts a substellar companion located at $\approx 44 \mathrm{AU}$, which has been claimed to be one of the lowest mass objects ever observed via direct imaging techniques (Kuzuhara et al. 2013). However, this conclusion relied on a young age for GJ 504, namely less than 200 Myr, as indicated by the rotational period of $P=3.33$ days. Conversely, Fuhrmann \& Chini (2015), thanks to high-resolution spectroscopic observations, found evidence for a much older age ( $\approx 3-6$ Gyr), suggesting that the substellar companion is actually a brown dwarf rather than a giant planet. In order to reconcile this old age, coming from isochrones and spectroscopy, with the indications of youth given by rotational and activity properties, these authors conceived the possibility of a merging event. The star GJ 504 might have engulfed a planetary-mass object that caused the spin up of rotational velocity along with enhancements in the chromospheric activity level. To shed light on this debated and complex picture, we exploited a strictly differential (line-by-line) analysis of GJ 504 with respect to two reference stars, namely $\iota$ Hor and HIP 84827. The role of $\iota$ Hor is particularly critical in this respect because its properties are very well constrained from an independent tool, i.e. asteroseismic observations (see Vauclair et al. 2008). Our results indicate that the surface gravity of GJ 504 is $0.2 \pm 0.07$ dex lower than that of the main-sequence star $\iota$ Hor, implying a slightly more advanced evolutionary stage for the object. The isochrone comparison provides us with an age range between 1.8 and $3.5 \mathrm{Gyr}$, which is qualitatively in agreement with Fuhrmann \& Chini results, although slightly younger. Interestingly, the solar Ba abundance also points to a very old age for the system, which is at variance with young stars in clusters and in the field (D'Orazi et al. 2009; Desidera et al. 2011) that are known to be characterised by an extremely high $\mathrm{Ba}$ content ( $[\mathrm{Ba} / \mathrm{Fe}]$ up to $0.6 \mathrm{dex})$. To investigate the merging scenario suggested by Fuhrmann \& Chini, we ran a tidal evolution code, assuming an age of roughly $\approx 2 \mathrm{Gyr}$ and imposing the condition that the engulfment event could not have occurred more than 200 Myr ago; otherwise the star would have lost the angular momentum and slowed down. Our tests indicate that a very plausible system architecture would result in an initial configuration of a planetary companion (with mass not larger than $\sim 3 M_{\mathrm{J}}$ ) located at $0.03 \mathrm{AU}$. This is probably because Kozai cycles (due to the presence of the external sub-stellar companion) have caused an inward migration. From such a small distance, the low-mass body has been affected by the stellar tides and slowly started to spiral down on the central star. If this were the case, we would expect to reveal planetary remnants such as rocky cores of the now defunct hot Jupiter in the proximity of star GJ 504. Current measurements prevent us from investigating this issue, but we plan to have purposely designed observations to detect such a small signal in radial velocity variations.

Acknowledgements. This work has made extensive use of the SIMBAD, Vizier, and NASA ADS databases. V.D. thanks Ivan Ramirez and Tamara Mishenina for very useful discussions and for providing information on unpublished material. We thank the anonymous referee for very helpful comments and suggestions.

\section{References}

Amarsi, A. M., Asplund, M., Collet, R., \& Leenaarts, J. 2015, MNRAS, 454, L11

Andrievsky, S. M., Spite, M., Korotin, S. A., et al. 2010, A\&A, 509, A88 Asplund, M., Grevesse, N., Sauval, A. J., \& Scott, P. 2009, ARA\&A, 47, 481

Baliunas, S., Sokoloff, D., \& Soon, W. 1996, ApJ, 457, L99

Battistini, C., \& Bensby, T. 2015, A\&A, 577, A9

Bensby, T., Feltzing, S., \& Oey, M. S. 2014, A\&A, 562, A71

Beuzit, J.-L., Feldt, M., Dohlen, K., et al. 2008, in Ground-based and Airborne Instrumentation for Astronomy II, Proc. SPIE, 7014, 701418

Biazzo, K., D’Orazi, V., Desidera, S., et al. 2012, MNRAS, 427, 2905

Bressan, A., Marigo, P., Girardi, L., et al. 2012, MNRAS, 427, 127

Bruls, J. H. M. J., Rutten, R. J., \& Shchukina, N. G. 1992, A\&A, 265, 237

Castelli, F., \& Kurucz, R. L. 2004, ArXiv e-prints

[arXiv: astro-ph/0405087]

De Silva, G. M., D’Orazi, V., Melo, C., et al. 2013, MNRAS, 431, 1005

Delgado Mena, E., Israelian, G., González Hernández, J. I., et al. 2014, A\&A, 562, A92

Desidera, S., Covino, E., Messina, S., et al. 2011, A\&A, 529, A54

Desidera, S., Covino, E., Messina, S., et al. 2015, A\&A, 573, A126 Donahue, R. A., Saar, S. H., \& Baliunas, S. L. 1996, ApJ, 466, 384 D'Orazi, V., \& Randich, S. 2009, A\&A, 501, 553

D’Orazi, V., Magrini, L., Randich, S., et al. 2009, ApJ, 693, L31

D'Orazi, V., Biazzo, K., Desidera, S., et al. 2012, MNRAS, 423, 2789

Findeisen, K., Hillenbrand, L., \& Soderblom, D. 2011, AJ, 142, 23

Fischer, D. A., Marcy, G. W., \& Spronck, J. F. P. 2014, ApJS, 210, 5

Fortney, J. J., Marley, M. S., Saumon, D., \& Lodders, K. 2008, ApJ, 683, 1104

Fuhrmann, K., \& Chini, R. 2015, ApJ, 806, 163

Gagné, J., Lafrenière, D., Doyon, R., Malo, L., \& Artigau, É. 2014, ApJ, 783, 121

Gonzalez, G., Carlson, M. K., \& Tobin, R. W. 2010, MNRAS, 403, 1368

Grevesse, N., Noels, A., \& Sauval, A. J. 1996, in Cosmic Abundances, eds. S. S. Holt, \& G. Sonneborn, ASP Conf. Ser., 99, 117

Ivanova, D. V., \& Shimanskii, V. V. 2000, Astron. Rep., 44, 376

Jackson, B., Jensen, E., Peacock, S., Arras, P., \& Penev, K. 2016, Celest. Mech. Dyn. Astron., 126, 227

Jacobson, H. R., \& Friel, E. D. 2013, AJ, 145, 107

Kaufer, A., Stahl, O., Tubbesing, S., et al. 1999, The Messenger, 95, 8

Korotin, S. A., Andrievsky, S. M., Hansen, C. J., et al. 2015, A\&A, 581, A70

Kratter, K. M., Murray-Clay, R. A., \& Youdin, A. N. 2010, ApJ, 710, 1375

Kuzuhara, M., Tamura, M., Kudo, T., et al. 2013, ApJ, 774, 11

Lockwood, G. W., Skiff, B. A., Henry, G. W., et al. 2007, ApJS, 171, 260

Lodders, K., Palme, H., \& Gail, H.-P. 2009, in Landolt Börnstein, Group VI, Vol. 4B

Macintosh, B., Graham, J. R., Ingraham, P., et al. 2014, PNAS, 111, 12661

Maldonado, J., Eiroa, C., Villaver, E., Montesinos, B., \& Mora, A. 2012, A\&A, 541, A40

Mamajek, E. E., \& Hillenbrand, L. A. 2008, ApJ, 687, 1264

Marley, M. S., Fortney, J. J., Hubickyj, O., Bodenheimer, P., \& Lissauer, J. J. 2007, ApJ, 655, 541

Marsakov, V. A., \& Shevelev, Y. G. 1995, Bulletin d'Information du Centre de Données Stellaires, 47, 13

Martinache, F., \& Guyon, O. 2009, in Techniques and Instrumentation for Detection of Exoplanets IV, Proc. SPIE, 7440, 744000

McWilliam, A., Preston, G. W., Sneden, C., \& Searle, L. 1995, AJ, 109, 2757

Meléndez, J., Ramírez, I., Karakas, A. I., et al. 2014, ApJ, 791, 14

Messina, S. 1998, Ph.D. Thesis, Catania Astrophysical Observatory, University of Catania, Italy

Messina, S., Monard, B., Worters, H. L., Bromage, G. E., \& Sanchez, R. Z. 2016, New Astron., 42, 29

Metcalfe, T. S., Basu, S., Henry, T. J., et al. 2010, ApJ, 723, L213

Mishenina, T. V., Pignatari, M., Korotin, S. A., et al. 2013, A\&A, 552, A128

Montes, D., López-Santiago, J., Gálvez, M. C., et al. 2001, MNRAS, 328, 45

Nidever, D. L., Marcy, G. W., Butler, R. P., Fischer, D. A., \& Vogt, S. S. 2002, ApJS, 141, 503

Pollack, J. B., Hubickyj, O., Bodenheimer, P., et al. 1996, Icarus, 124, 62 
V. D'Orazi et al.: A critical reassessment of the fundamental properties of GJ 504: chemical composition and age

Ramírez, I., Allende Prieto, C., \& Lambert, D. L. 2013, ApJ, 764, 78

Ramírez, I., Meléndez, J., \& Asplund, M. 2014, A\&A, 561, A7

Reggiani, M., Meyer, M. R., Chauvin, G., et al. 2016, A\&A, 586, A147

Santos, N. C., Melo, C., James, D. J., et al. 2008, A\&A, 480, 889

Sestito, P., \& Randich, S. 2005, A\&A, 442, 615

Sestito, P., Randich, S., \& Pallavicini, R. 2004, A\&A, 426, 809

Skemer, A. J., Morley, C. V., Zimmerman, N. T., et al. 2016, ApJ, 817, 166

Sneden, C. A. 1973, Ph.D. Thesis, the University of Texas at Austin, USA

Soderblom, D. R., Jones, B. F., Balachandran, S., et al. 1993, AJ, 106, 1059

Sousa, S. G., Santos, N. C., Israelian, G., Mayor, M., \& Monteiro, M. J. P. F. G. 2007, A\&A, 469, 783
Takeda, Y., \& Kawanomoto, S. 2005, PASJ, 57, 45

Thorburn, J. A., Hobbs, L. M., Deliyannis, C. P., \& Pinsonneault, M. H. 1993, ApJ, 415, 150

Valenti, J. A., \& Fischer, D. A. 2005, ApJS, 159, 141

Valsecchi, F., Rasio, F. A., \& Steffen, J. H. 2014, ApJ, 793, L3

Valsecchi, F., Rappaport, S., Rasio, F. A., Marchant, P., \& Rogers, L. A. 2015, ApJ, 813, 101

Vauclair, S., Laymand, M., Bouchy, F., et al. 2008, A\&A, 482, L5

Vigan, A., Bonnefoy, M., Ginski, C., et al. 2016, A\&A, 587, A55

Zahn, J.-P. 2008, in EAS Publ. Ser. 29, eds. M.-J. Goupil, \& J.-P. Zahn, 67

Zurlo, A., Vigan, A., Hagelberg, J., et al. 2013, A\&A, 554, A21 